\title{
ON SOME REVERSE INTEGRAL INEQUALITIES
}

\section{LUCIANA NANIA}

(Received 14 March 1989; revised 5 June 1989)

Communicated by Brailey Sims

\begin{abstract}
We prove the higher integrability of nonnegative decreasing functions, verifying a reverse inequality, and we calculate the optimal integrability exponent for these functions.
\end{abstract}

1980 Mathematics subject classification (Amer. Math. Soc.) (1985 Revision): 26 D 15.

\section{Introduction}

The aim of this paper is to prove

Theorem 1.1. Let $q>1, C>1$ and $g:(0, m) \rightarrow(0,+\infty)$ be a decreasing function satisfying

$\frac{1}{b-a} \int_{a}^{b} g^{q}(x) d x \leq C g^{q-1}(b) \frac{1}{b-a} \int_{a}^{b} g(x) d x \quad$ for all $(a, b) \subseteq(0, m)$.

Then, for every $p \in[q,(C q-1) /(C-1))$ the function $g$ belongs to $L^{p}(0, m)$ and there exists a constant $K=K(C, p, q)$ such that

$$
\frac{1}{b-a} \int_{a}^{b} g^{p}(x) d x \leq K\left(\frac{1}{b-a} \int_{a}^{b} g(x) d x\right)^{p} \quad \text { for all }(a, b) \subseteq(0, m),
$$

with $K(C, p, q) \rightarrow \infty$ as $p \rightarrow(C q-1) /(C-1)$. The result is sharp.

This work has been performed under a national research program of Italian M.P.I. (40\%-1986).

(C) 1990 Australian Mathematical Society 0263-6115/90 \$A2.00+0.00 
Since for every decreasing function $g(x)$ we have

$$
\frac{1}{b-a} \int_{a}^{b} g^{q}(x) d x \geq \frac{g^{q-1}(b)}{b-a} \int_{a}^{b} g(x) d x
$$

the inequality (1.1) is a reverse inequality, that is, an inequality which is the inverse of (1.3) but has a constant greater than 1 on the right side. Inequality (1.1) appears, for example, in the paper of Gehring [2], in his remarkable theorem showing higher integrability of a function verifying a reverse Hölder inequality. The upper bound for $p$ is sharp since the function $g(x)=x^{-\beta}$, with $\beta=(C-1) /(C q-1)$ satisfies (1.1) but fails to belong to $L^{p}(0, m)$ with $p=(C q-1) /(C-1)$.

Theorem (1.1) is related to papers [4] and [6], where some sharp results are proved relative to two different reverse inequalities (see, respectively, Lemma 2.3 and Lemma 2.4 of the present paper).

\section{Preliminaries}

We begin with the classical Hardy's inequality [3].

LemMA 2.1. Let $g=g(t)$ belong to $L^{q}(0, m), q>1$. Then

$$
\int_{0}^{m}\left|\frac{1}{t} \int_{0}^{t} g(s) d s\right|^{q} d t \leq\left(\frac{q}{q-1}\right)^{q} \int_{0}^{m}|g(t)|^{q} d t .
$$

In the sequel, we shall use the weighted Hardy's inequality [3]. Then

LEMMA 2.2. Let $f \geq 0, F(x)=\frac{1}{x} \int_{0}^{x} f(t) d t, q>1$, and $\alpha<q-1$.

$$
\int_{0}^{m} x^{\alpha} F^{q}(x) d x \leq\left(\frac{q}{q-1-\alpha}\right)^{q} \int_{0}^{m} x^{\alpha} f^{q}(x) d x .
$$

Let us now recall a sharp higher integrability result proved by Muckenhoupt [4].

LEMMA 2.3. Let $h=h(t)$ be positive and decreasing on $(0, m)$ and assume that there exists $A>1$ such that

$$
\frac{1}{t} \int_{0}^{t} h(s) d s \leq A h(t) \quad \text { for all } t \in(0, m) .
$$

Then, for every $r \in[1, A /(A-1))$, the function $h$ belongs to $L^{r}(0, m)$ and

$$
\frac{1}{m} \int_{0}^{m} h^{r}(s) d s \leq \frac{A}{A-r(A-1)}\left(\frac{1}{m} \int_{0}^{m} h(s) d s\right)^{r} \text {. }
$$


The result is sharp, because the function $f(x)=x^{-\beta}$ with $\beta=(A-1) / A$ satisfies (2.1) but fails to belong to $L^{r}(0, m)$ with $r=A /(A-1)$.

The following sharp result is proved in [6].

Lemma 2.4. Let $B>1$, and let $f:(0, m) \rightarrow(0,+\infty)$ be a decreasing function satisfying

$$
\frac{1}{b-a} \int_{a}^{b} f^{2}(x) d x \leq B\left(\frac{1}{b-a} \int_{a}^{b} f(x) d x\right)^{2} \quad \text { for all }(a, b) \subseteq(0, m) .
$$

Set $\varepsilon(B)=\sqrt{B /(B-1)}-1$. Then the function $f$ belongs to $L^{p}(a, b)$ for $p \in[2,2+\varepsilon)$ and there exists a constant $c=c(p, B)$ such that

$$
\frac{1}{b-a} \int_{a}^{b} f^{p}(x) d x \leq c\left(\frac{1}{b-a} \int_{a}^{b} f(x) d x\right)^{p}
$$

with $c(p, B) \rightarrow \infty$ as $p \rightarrow 2+\varepsilon(B)$. The result is sharp, because the function $f(x)=x^{-\beta}$ with $\beta=1-B+\sqrt{B^{2}-B}$ satisfies (2.3) but fails to belong to $L^{p}(a, b)$ with $p=2+\varepsilon(B)$.

Finally, we quote another result from [3].

LEMMA 2.5. Let $f:(0, m) \rightarrow(0,+\infty)$ be a decreasing function. Then for $-1<\beta<0,(a, b) \subseteq(0, m), q>1$

$$
\left(\int_{a}^{b} f^{q /(1+\beta)} d t\right)^{1+\beta} \leq \int_{a}^{b}(t-a)^{\beta} f^{q}(t) d t .
$$

\section{A first result of higher integrability}

Let $g:(0, m] \rightarrow(0,+\infty)$ be a nonnegative decreasing function. Then the following inequality holds.

$$
\frac{g^{q-1}(t)}{t} \int_{0}^{t} g(x) d x \leq \frac{1}{t} \int_{0}^{t} g^{q}(x) d x
$$

for any $t \in(0, m]$ and for any $q>1$.

The following theorem shows that if $g$ satisfies an inequality which is reverse to (3.1), then $g$ has higher integrability. 
TheOREM 3.1. Let $C>1, q>1$. Then there exists a positive $\varepsilon=$ $\varepsilon(q, C)$, such that every positive decreasing function $g$ satisfying

$$
\frac{1}{t} \int_{0}^{t} g^{q}(x) d x \leq \frac{C g^{q-1}(t)}{t} \int_{0}^{t} g(x) d x \quad \text { for all } t \in(0, m]
$$

belongs to $L^{p}(0, m)$ for $p \in[q, q+\varepsilon]$, and we have

$$
\frac{1}{m} \int_{0}^{m} g^{p}(x) d x \leq \frac{(C q)^{(p+q) / q}}{(q-1)^{p / q}[C(q-p)+p(1 / q-1)]}\left(\frac{1}{m} \int_{0}^{m} g^{q}(x) d x\right)^{p / q} .
$$

Proof. Let us integrate (3.1) between 0 and $y \in(0, m]$; using the SchwarzHölder inequality and Lemma 2.1 , we have

$$
\begin{aligned}
\int_{0}^{y} \frac{1}{t} \int_{0}^{t} g^{q}(x) d x d t \leq C \int_{0}^{y} \frac{g^{q-1}(t)}{t} \int_{0}^{t} g(x) d x d t \\
\quad \leq C\left(\int_{0}^{y}\left(g^{q-1}(t)\right)^{q /(q-1)} d t\right)^{(q-1) / q}\left(\int_{0}^{y}\left(\frac{1}{t} \int_{0}^{t} g(x) d x\right)^{q} d t\right)^{1 / q} \\
\quad \leq C \frac{q}{q-1}\left(\int_{0}^{y} g^{q}(x) d x\right)^{(q-1) / q}\left(\int_{0}^{y} g^{q}(x) d x\right)^{1 / q} .
\end{aligned}
$$

Therefore, if we set $h(t)=\frac{1}{t} \int_{0}^{t} g^{q}(x) d x$, we have proved that

$$
\frac{1}{y} \int_{0}^{y} h(t) d t \leq C\left(\frac{q}{q-1}\right) h(y) \text { for all } y \in(0, m] .
$$

We can now apply Lemma 2.3 to $h(t)$ and we get

$$
\begin{aligned}
\frac{1}{m} \int_{0}^{m} h^{r}(t) d t \leq \frac{C q}{C q-r(C q-q+1)}\left(\frac{1}{m} \int_{0}^{m} h(t) d t\right)^{r} & \\
r & \in\left[1, \frac{C q}{C q-q+1}\right) .
\end{aligned}
$$

Since $g(x)$ is decreasing, we have $g^{q}(t) \leq \frac{1}{t} \int_{0}^{t} g^{q}(x) d x$ and it follows from (3.5) that

$$
\frac{1}{m} \int_{0}^{m} g^{r q}(t) d t \leq \frac{C q}{C q-r(C q-q+1)}\left(\frac{1}{m} \int_{0}^{m} h(t) d t\right)^{r} .
$$

Since

we finally obtain

$$
\frac{1}{m} \int_{0}^{m} h(t) d t \leq \frac{C q}{(q-1) m} \int_{0}^{m} g^{q}(t) d t
$$
(3.7)
$\frac{1}{m} \int_{0}^{m} g^{r q}(t) d t \leq \frac{(C q)^{r+1}}{(q-1)^{r}[C q-r(C q-q+1)]}\left(\frac{1}{m} \int_{0}^{m} g^{q}(t) d t\right)^{r}$ 
as desired. This higher integrability result could also be deduced from that proved in [5, Proposition 1], from the fact that $g$ is decreasing; however, the constant in (3.2) is better than that obtained in [5].

\section{The integrability exponent}

We shall now calculate the "optimal" integrability exponent $p(C, q)$ of a function satisfying the assumptions of Theorem 3.1. With regard to this, we recall that in [6] the best integrability exponent has been calculated for a function $g$ satisfying the reverse Hölder inequality

$$
\frac{1}{b-a} \int_{a}^{b} g^{2} d x \leq B\left(\frac{1}{b-a} \int_{a}^{b} g d x\right)^{2}
$$

(see Lemma 2.4).

Since $g$ is decreasing, inequality (3.1) for $q=2$ implies (4.1) with the same constant $C$. Therefore, we can expect for the functions verifying (3.1) a value of the optimal exponent higher than that one calculated in [6]. Moreover, we are able to calculate this exponent also in the case $q>2$.

With this aim, we state the following Hardy type inequality.

Lemma 4.1. Let $g:(a, b) \rightarrow(0,+\infty), q>1, \alpha<q-1$. Then,

$$
\int_{a}^{b}(x-a)^{\alpha} g^{q-1}(x)\left(\frac{1}{x-a} \int_{a}^{x} g(s) d s\right) d x \leq \frac{q}{q-1-\alpha} \int_{a}^{b}(x-a)^{\alpha} g^{q}(x) d x .
$$

Proof. Using the Schwarz-Hölder inequality and Lemma 2.2 we get

$$
\begin{aligned}
\int_{a}^{b}(x-a)^{\alpha} g^{q-1}(x)\left(\frac{1}{x-a} \int_{a}^{x} g(s) d s\right) d x \\
=\int_{a}^{b}(x-a)^{(q-1) \alpha / q}(x-a)^{\alpha / q} g^{q-1}(x)\left(\frac{1}{x-a} \int_{a}^{x} g d s\right) d x \\
\leq\left(\int_{a}^{b}\left((x-a)^{(q-1) \alpha / q} g^{q-1}(x)\right)^{q /(q-1)} d x\right)^{(q-1) / q} \\
\quad \times\left(\int_{a}^{b}\left((x-a)^{\alpha / q}\left(\frac{1}{x-a} \int_{a}^{x} g d s\right)\right)^{q} d x\right)^{1 / q} \\
\leq \frac{q}{q-1-\alpha} \int_{a}^{b}(x-a)^{\alpha} g^{q}(x) d x,
\end{aligned}
$$

that is, we have (4.2). 
LEMMA 4.2. Let $g:(0, m) \rightarrow(0,+\infty)$ satisfy

$$
\frac{1}{b-a} \int_{a}^{b} g^{q}(x) d x \leq \frac{C g^{q-1}(b)}{b-a} \int_{a}^{b} g(x) d x \quad \text { for all }(a, b) \subset(0, m)
$$

and $g \in L^{\infty}(0, m)$. Set $\gamma(\alpha)=1+\alpha C q /(q-1-\alpha)$, for $1-q /(C q-1)<$ $\alpha<0$. Then

$\gamma(\alpha) \int_{a}^{b}(x-a)^{\alpha} g^{q}(x) d x \leq(b-a)^{\alpha} \int_{a}^{b} g^{q}(x) d x \quad$ for all $(a, b) \subset(0, m)$.

Proof. By (4.3), using the Fubini theorem, we get

$$
\begin{aligned}
\int_{a}^{b}(x-a)^{\alpha} g^{q-1}(x)\left(\frac{1}{x-a} \int_{a}^{x} g(t) d t\right) d x \\
\quad \geq \frac{1}{C} \int_{a}^{b}(x-a)^{\alpha-1} \int_{a}^{x} g^{q}(t) d t d x \\
=\frac{1}{C} \int_{a}^{b} g^{q}(t) \int_{t}^{b}(x-a)^{\alpha-1} d x d t \\
=\frac{1}{\alpha C} \int_{a}^{b} g^{q}(t)\left[(b-a)^{\alpha}-(t-a)^{\alpha}\right] d t
\end{aligned}
$$

By Lemma (4.1), from (4.5) it follows that

$$
\frac{q}{q-1-\alpha} \int_{a}^{b}(x-a)^{\alpha} g^{q}(x) d x \geq \frac{1}{\alpha C} \int_{a}^{b} g^{q}(x)\left[(b-a)^{\alpha}-(x-a)^{\alpha}\right] d x,
$$

and from this, since $\alpha<0$, we deduce

$$
\left(\frac{C q}{q-1-\alpha}+1\right) \int_{a}^{b}(x-a)^{\alpha} g^{q}(x) d x \leq(b-a)^{\alpha} \int_{a}^{b} g^{q}(x) d x .
$$

We eliminate the assumption $g \in L^{\infty}(0, m)$ in Lemma 4.2, by an approximation argument. First, we prove the following

LEMMA 4.3. Let $g=g(t)$ be a non-negative, decreasing function satisfying

$$
\int_{a}^{b} g^{q}(x) d x \leq C g^{q-1}(b) \int_{a}^{b} g(x) d x \quad \text { for all }(a, b) \subset(0, m) .
$$

Then the convolution $G(x)=\eta * g(x)=\int_{R} \eta(y) g(x-y) d y$ of $g$ with a mollifier $\eta$ satisfies (4.6) with the same constant $C$. 
Proof. Let $x-y=z$; then, from (4.6) we get

$$
\begin{gathered}
\int_{a}^{b} g^{q}(x-y) d x=\int_{a-y}^{b-y} g^{q}(z) d z \\
\leq C g^{q-1}(b-y) \int_{a-y}^{b-y} g(z) d z \\
\quad=C g^{q-1}(b-y) \int_{a}^{b} g(x-y) d x .
\end{gathered}
$$

Moreover, from [1] we have

$$
\left(\int_{R} G^{q}(x) d x\right)^{1 / q} \leq \int_{R} \eta(y)\left(\int_{a}^{b} g^{q}(x-y) d x\right)^{1 / q} d y
$$

From (4.7), (4.8) and the Schwarz-Hölder inequality it follows that

$$
\begin{aligned}
& \left(\int_{a}^{b} G^{q}(x) d x\right)^{1 / q} \\
& \quad \leq C^{1 / q} \int_{R} \eta(y)\left(g^{q-1}(b-y) \int_{a}^{b} g(x-y) d x\right)^{1 / q} d y \\
& \quad=C^{1 / q} \int_{R} \eta^{q-1 / q}(y) \eta^{1 / q}(y)\left(g^{q-1}(b-y) \int_{a}^{b} g(x-y) d x\right)^{1 / q} d y \\
& \quad \leq C^{1 / q}\left(\int_{R} \eta(y) g(b-y) d y\right)^{q-1 / q}\left(\int_{R} \eta(y) \int_{a}^{b} g(x-y) d x d y\right)^{1 / q},
\end{aligned}
$$

and from this the result follows.

Proof of Theorem 1.1. We can write (4.4) for $G_{h}=\eta_{h} * g$ and, passing to limit as $h \rightarrow \infty$, we get

$$
\gamma(\alpha) \int_{a}^{b}(x-a)^{\alpha} g^{q}(x) d x \leq(b-a)^{\alpha} \int_{a}^{b} g^{q}(x) d x .
$$

From this and Lemma (2.5) it follows that

$$
\left(\int_{a}^{b} g^{q / 1+\alpha}(x) d x\right)^{1+\alpha} \leq \frac{(b-a)^{\alpha}}{\gamma(\alpha)} \int_{a}^{b} g^{q}(x) d x \text { for } \alpha>\frac{1-q}{C q-1} .
$$

In conclusion, we have obtained that $g \in L^{p}(0, m)$ with $q \leq p<$ $(C q-1) /(C-1)$. The function $g(x)=x^{-\beta}$ with $\beta=(C-1) /(C q-1)$ satisfies $(1.1)$ but fails to belong to $L^{p}(0, m)$ for $p=(C q-1) /(C-1)$ and so the result is sharp. 


\section{References}

[1] B. Bojarski and T. Iwaniec, 'Analytical foundations of the theory of quasi conformal mapping in $R^{n}$, Ann. Acad. Sci. Fenn. Ser. AI Math. 8 (1983), 257-324.

[2] F. W. Gehring, 'The $L^{p}$ integrability of the partial derivatives of a quasi conformal mapping', Acta Math. 130 (1973), 265-277.

[3] G. H. Hardy, J. E. Littlewood and G. Polya, Inequalities, (Cambridge Univ. Press, 1964).

[4] B. Muckenhoupt, 'Weighted norm inequalities for the Hardy maximal function', Trans. Amer. Math. Soc. 165 (1972), 207-226.

[5] C. Sbordone, 'Higher integrability from reverse integral inequalities', Proc. Internat. Meeting-Methods of Functional Analysis and Theory of Elliptic Equations, (Napoli, Liguori, 1983).

[6] C. Sbordone, 'Rearrangement of functions and reverse Hölder inequalities', Ennio de Giorgi Colloqium, pp. 134-146, Paris, 1983 (Pitman Res. Notes in Math. 125, 1985).

\section{Dipartimento di Matematica e Applicazioni}

"R. Caccioppoli"

Via Mezzocannone 8, 80134 Napoli

Italy 